12,09

\title{
Перестройка спектров электролюминесценции в гетероструктурах II типа $n$-InAs/n-InAsSbP
}

\author{
() В.В. Романов, Э.В. Иванов, К.Д. Моисеев \\ Физико-технический институт им. А.Ф. Иофрфе, \\ Санкт-Петербург, Россия \\ ฯ E-mail: mkd@iropt2.ioffe.rssi.ru
}

(Поступила в Редакцию 20 сентября 2017 г.)

\begin{abstract}
Методом МОГФЭ были получены одиночные гетеросруктуры II типа $n^{+}-\operatorname{InAs} / n^{0}-\operatorname{InAs}_{0.59} \mathrm{Sb}_{0.16} \mathrm{P}_{0.25}$ на основе преднамеренно нелегированного эпитаксиального слоя с электронным типом проводимости. При этом вблизи гетерограницы в объеме четверного твердого раствора был сформирован переходной слой модулированного состава. Было показано существование канала излучательной рекомбинации, обусловленного наличием локализованных дырочных состояний в квантовых ямах, сформированных в переходном слое вблизи гетерограницы. Была продемонстрирована перестройка максимума интенсивности спектра ЭЛ исследуемой гетероструктуры при приложении прямого внешнего смещения. Результаты настоящего исследования могут быть использованы при разработке перестраиваемых светодиодов, работающих в среднем инфракрасном диапазоне $2-4 \mu \mathrm{m}$.
\end{abstract}

DOI: $10.21883 /$ FTT.2018.03.45565.268

\section{1. Введение}

При наращивании эпитаксиальных слоев в системе твердых растворов In-As-Sb-P на подложку InAs методом газофазной эпитаксии из металлоорганических соединений (МОГФЭ) было обнаружено модулированное изменение состава твердого раствора вблизи гетерограницы подложка/слой в интервале толщин 50-100 nm (так называемый переходной слой) [1]. Модуляция изменения состава в переходном слое вдоль направления эпитаксиального роста заключалась в появлении колебаний концентраций компонентов, входящих в твердый раствор. Было экспериментально установлено, что изменение концентрации фосфора и сурьмы происходит синхронно и обусловлено эффектом самоорганизации изоморфных твердых растворов InAsSbP, обогащенных арсенидом индия [2]. Следует отметить, что степень самоорганизации твердой фазы наращиваемого слоя определялась выбранной величиной соотношения в газовой фазе $\mathrm{TMSb} / \mathrm{PH}_{3}$, которая поддерживалась постоянной в процессе эпитаксии. При этом концентрация радикалов соединений мышьяка в газовой фазе определяла величину градиента концентрации компонентов для каждого заданного твердого раствора $\operatorname{InAs}_{1-x-y} \mathrm{Sb}_{y} \mathrm{P}_{x}$. Наличие переходного слоя толщиной в несколько десятков нанометров с модулированным составом может стать основанием для формирования квантово-размерных объектов вблизи гетерограницы, которые образуют дополнительную область локализации носителей заряда в гетероструктуре. Появление дополнительных каналов излучательной рекомбинации является существенным фактором, влияющим на работу оптоэлектронных приборов, и должно быть учтено при моделировании гетероструктур на основе данной системы твердых растворов. В настоящей работе мы изучали электрические и люминесцентные свойства одиночного гетероперехода II типа $n$-InAs/ $n$-InAsSbP, сформированного полупроводниками с одним типом проводимости и содержащего модулированный по составу переходной слой вблизи гетерограницы.

\section{2. Технология изготовления структур и методика экспериментов}

Одиночные изотипные гетероструктуры $n^{+}$-InAs/ $n^{0}$-InAsSbP были получены методом МОГФЭ путем наращивания четверного твердого раствора $\mathrm{InAs}_{0.59} \mathrm{Sb}_{0.16} \mathrm{P}_{0.25}$ на подложке InAs c рабочей поверхностью, ориентированной вдоль кристаллографической оси (001). Подложка InAs была легирована донорной примесью (серой) до концентрации свободных электронов $n=2 \cdot 10^{18} \mathrm{~cm}^{-3}$. Наращивание слоя твердого раствора InAsSbP проводилось при атмосферном давлении и температуре $510^{\circ} \mathrm{C}$. В качестве источников применялись следующие металлоорганические соединения: триметилиндий (TMIn), триметилстибин (TMSb), третбутиларсин (TBAs). Источником фосфора служил фосфин $\left(\mathrm{PH}_{3}\right)$, разбавленный в водороде до концентрации в $20 \%$. Особенности технологии наращивания данных твердых растворов описаны ранее в [2]. В процессе эпитаксии твердый раствор InAsSbP преднамеренно не легировался, и концентрация электронов в нем не превышала $n=6 \cdot 10^{16} \mathrm{~cm}^{-3}$. Толщина слоя составляла примерно $1 \mu \mathrm{m}$. Относительное рассогласование постоянных кристаллических решеток между подложкой InAs и эпитаксиальным слоем $\mathrm{InAs}_{0.59} \mathrm{Sb}_{0.16} \mathrm{P}_{0.25}$ не превышало величины $+0.3 \%$.

Для изучения электрических и люминесцентных характеристик полученных образцов были изготовлены 
меза-диодные структуры с помощью стандартной методики фотолитографического совмещения с применением метода мокрого травления. Диаметр мезы составлял $500 \mu \mathrm{m}$, размер точечного контакта - $50 \mu \mathrm{m}$. Исследуемые меза-диоды были смонтированы на держателях ТО-18. Спектральные характеристики полученных гетероструктур исследовались с помощью автоматизированной установки на базе монохроматора DK-480 с дифракционной решеткой 150 штр/mm (CVI Laser Corp, USA), селективного усилителя SR-810 (Stanford Research Systems, USA) и фото-вольтаического детектора на основе антимонида индия, работающего при температуре жидкого азота (Judson Co, USA). Возбуждение электролюминесценции (ЭЛ) осуществлялось прямоугольными импульсами тока с коэффициентом заполнения 50\% и частотой повторения $512 \mathrm{~Hz}$.

\section{3. Электрические и люминесцентные свойства изотипного гетероперехода $n^{+}-\operatorname{InAs} / \boldsymbol{n}^{0}-\operatorname{InAsSbP}$ при комнатной температуре}

На рис. 1 представлены вольтамперные характеристики (BAX) гетероперехода $n^{+}-\mathrm{InAs} / n^{0}$-InAsSbP, полученные при комнатной температуре $(300 \mathrm{~K})$ и при температуре жидкого азота $(77 \mathrm{~K})$. Как видно из рисунка, ВАХ для обеих температур демонстрируют ярко выраженную асимметричную зависимость тока, протекающего через гетероструктуру, от внешнего смещения. Следует подчеркнуть, что прямым смещением мы считаем такую полярность приложенного напряжения, когда отрицательный потенциал прикладывается к подложке $n^{+}-\mathrm{InAs}$, тогда как положительный - к эпитаксиальному слою $n$-InAsSbP. Следовательно, обратное смещение соответствует противоположной комбинации приложенных потенциалов. Полученные в данной работе зависимости похожи на характеристики обращенного диода. Более того, в области обратных смещений обнаружена практически линейная зависимость тока от величины прикладываемого напряжения, которая почти не зависела от температуры окружающей среды. Подобное поведение BAX наблюдалось ранее для гетероструктур II типа $n^{+}-\mathrm{Si} / n-\mathrm{Ge}[3]$.

Как видно из рис. 1, на начальном участке прямой ветви ВАХ существует область насыщения по току в интервале прикладываемых напряжений 20-180 mV (300 K) и $20-400 \mathrm{mV}(77 \mathrm{~K})$. В этой области ток, протекающий через гетероструктуру, слабо возрастает от 5 до $12 \mathrm{~mA}$ по мере увеличения приложенного напряжения. При дальнейшем повышении прямого смещения наблюдается резкий рост величины тока, и ВАХ демонстрируют линейные зависимости с наклонами, соответствующими последовательному сопротивлению $R_{S} \sim 0.75 \Omega$ для $T=300 \mathrm{~K}$ и $0.42 \Omega$ для $T=77 \mathrm{~K}$. Причиной такого влияния температуры является увеличение сопротивления объемной части гетероструктуры, которое возрастает

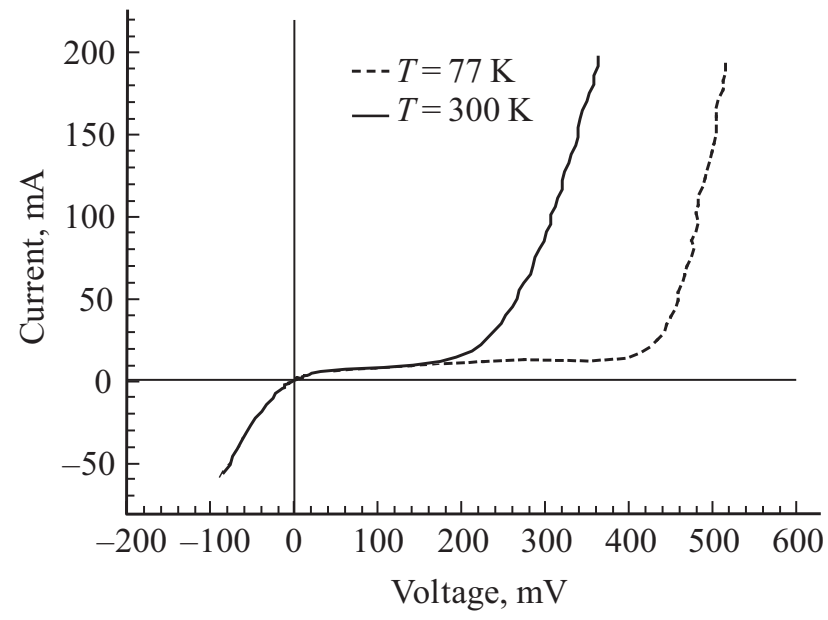

Рис. 1. Вольтамперные характеристики изотипного гетероперехода $n^{+}$-InAs $/ n^{0}$-InAsSbP при $300 \mathrm{~K}$ (сплошная линия) и $77 \mathrm{~K}$ (пунктирная линия).

при повышении температуры вследствие снижения подвижности свободных носителей заряда. Значения напряжения порога проводимости определялись как точки пересечения касательной к линейному участку прямой ветви ВАХ с осью абсцисс. Значение данного параметра при комнатной температуре $U_{300 \mathrm{~K}}=0.22 \mathrm{~V}$ может быть ассоциировано с величиной порядка 2/3 ширины запрещенной зоны InAs. Рассчитанная таким способом величина $E_{G}^{300}=0.33 \mathrm{eV}$ несколько отличается от табличного значения $0.356 \mathrm{eV}$ [4]. Полученная разница может быть обусловлена высокой концентрацией примесных атомов, при которой донорная зона „размывается“ настолько, что смыкается с зоной проводимости [5]. После смыкания зон нижний уровень донорной зоны выполняет роль дна зоны проводимости, и эффективная ширина запрещенной зоны полупроводника уменьшается. В свою очередь величина $U_{77 \mathrm{~K}}=0.43 \mathrm{~V}$ может быть результатом суперпозиции вкладов последовательных сопротивлений: эпитаксиального слоя InAsSbP и переходного слоя на гетерогранице. При этом обратная ветвь ВАХ практически не зависела от температуры окружающей среды, что можно объяснить снижением потенциальных барьеров на гетерогранице II типа и увеличением энергии подвижных носителей зарядов при высокой концентрации электронов в $n^{+}$-InAs.

На рис. 2 представлены спектры ЭЛ, полученные при комнатной температуре для токов инжекции в интервале 20-200 mА при прямой полярности приложенного смещения. Из рисунка видно, что каждый спектр ЭЛ содержит две полосы излучения. Наиболее интенсивная полоса излучения $\left(h v_{1}\right)$ имеет симметричную форму с полушириной $50 \mathrm{meV}$ и демонстрирует максимум интенсивности в области энергий фотона $0.338-0.343 \mathrm{eV}$, что соответствует межзонным излучательным переходам в $n^{+}$-InAs. C увеличением тока накачки полуширина полосы $h v_{1}$ не изменяется, а максимум смещается на $5 \mathrm{meV}$ в область больших энергий фотона, что обуслов- 


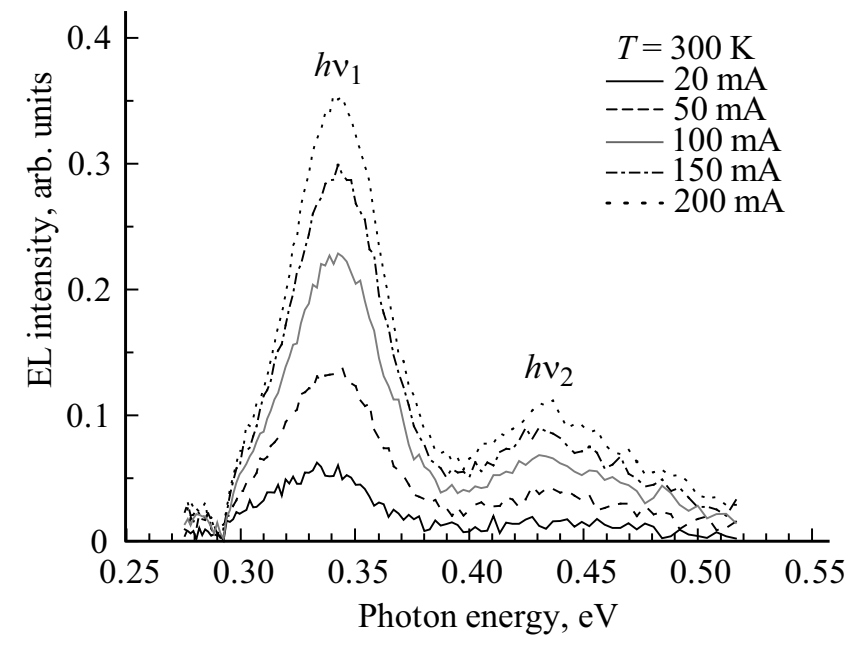

Рис. 2. Спектры ЭЛ в гетероструктуре $n^{+}$-InAs/ $n^{0}$-InAs ${ }_{0.59} \mathrm{Sb}_{0.16} \mathrm{P}_{0.25}$ при различных токах накачки для случая прямого смещения при температуре $300 \mathrm{~K}$.

лено эффектом Бурштейна-Мосса для высоких уровней накачки. Увеличение тока инжекции также приводило к появлению и дальнейшему возрастанию интенсивности полосы излучения $h v_{2}$, максимум которой находился в области энергий фотона $0.435 \mathrm{eV}$. Данная величина может быть приписана значению ширины запрещенной зоны слоя четверного твердого раствора $\operatorname{InAs}_{0.59} \mathrm{Sb}_{0.16} \mathrm{P}_{0.25}$. Следует отметить, что при обратной полярности приложенного к гетероструктуре внешнего смещения ЭЛ отсутствовала.

\section{4. Особенности люминесцентных свойств изотипного гетероперехода $n^{+}-\ln A s / n^{0}-\ln A s S b P$ при низких температурах}

При температуре $T=77 \mathrm{~K}$ спектры ЭЛ при прямой полярности приложенного смещения демонстрировали одну явно выраженную полосу излучения (рис. 3). При этом форма спектра для всех токов накачки была несимметрична, с резким высокоэнергетическим краем и более пологим низкоэнергетическим. Основная полоса излучения с полушириной порядка $30 \mathrm{meV}$ возникала в окрестности $0.43 \mathrm{eV}$, тогда как в области более низких энергий фотонов $(0.35-0.41 \mathrm{eV})$ наблюдался сильно затянутый „хвост“ спектрального излучения, который можно ассоциировать с другой более слабой и широкой (полуширина $\sim 60 \mathrm{meV}$ ) полосой. С увеличением тока накачки максимум более интенсивной полосы при сохранении полуширины пика ЭЛ 0.30-0.41 meV смещался в сторону больших энергий фотонов от 0.43 до $0.47 \mathrm{eV}$, что является весьма значительным сдвигом „голубым“ для одиночной гетероструктуры. Следует отметить, что интенсивность полосы излучения в области энергий фотонов вблизи $0.41 \mathrm{eV}$ менялась слабо при начальных значе- ниях тока накачки (20-50 mA) и достигала насыщения при более высоких значениях (50-100 mA). При этом ощутимого изменения ширины данной полосы не наблюдалось, она колебалась в интервале 60-65 meV. Разница в значениях полуширин наблюдаемых полос излучения говорит о существовании нескольких параллельных механизмов излучательной рекомбинации в исследуемой гетероструктуре $n^{+}-\mathrm{InAs} / n^{0}-\mathrm{InAs}_{0.59} \mathrm{Sb}_{0.16} \mathrm{P}_{0.25}$. При обратной полярности приложенного к гетероструктуре внешнего напряжения ЭЛ также отсутствовала как и при комнатной температуре.

На рис. 4 представлены варианты аппроксимации экспериментально полученных спектров ЭЛ несколькими кривыми в форме распределений Гаусса. Как видно из рисунка, на пороге регистрации излучения из одиночной гетероструктуры $n^{+}$-InAs $/ n^{0}$-InAsSbP при токе накачки $I=20 \mathrm{~mA}$ наблюдались две полосы излучения: $h v_{A}=0.41 \mathrm{eV}$ и $h v_{B}=0.43 \mathrm{eV}$ (рис. 4,a). При увеличении тока до $I=30 \mathrm{~mA}$ на высокоэнергетическом крае появляется третья полоса излучения $h v_{C}=0.465 \mathrm{meV}$ (см. рис. 4,b). Спектральное положения полосы $h v_{C}$ может быть приписано межзонным излучательным переходам в объеме эпитаксиального слоя $\operatorname{InAs}_{0.59} \mathrm{Sb}_{0.16} \mathrm{P}_{0.25}$. Согласно рис. 3 при высоких уровнях накачки $(I>50 \mathrm{~mA})$ полоса излучения $h v_{C}$ становится доминирующей в спектрах ЭЛ при $77 \mathrm{~K}$. Следует отметить, что с ростом накачки положение максимума полосы $h v_{C}$ изменялось незначительно (сдвиг по энергии составил порядка $5 \mathrm{meV}$ ), что также может быть объяснено проявлением эффекта Бурштейна-Мосса при высоком уровне инжекции носителей в область рекомбинационных переходов. Специфические особенности (провалы) на спектрах ЭЛ при высоких уровнях накачки обусловлены поглощением оптического излучения молекулами газов, присутствующих в лабораторной атмосфере: $2.60 \mu \mathrm{m}, 2.68 \mu \mathrm{m}, 2.78 \mu \mathrm{m}$ - пары воды $\left(\mathrm{H}_{2} \mathrm{O}\right)$,

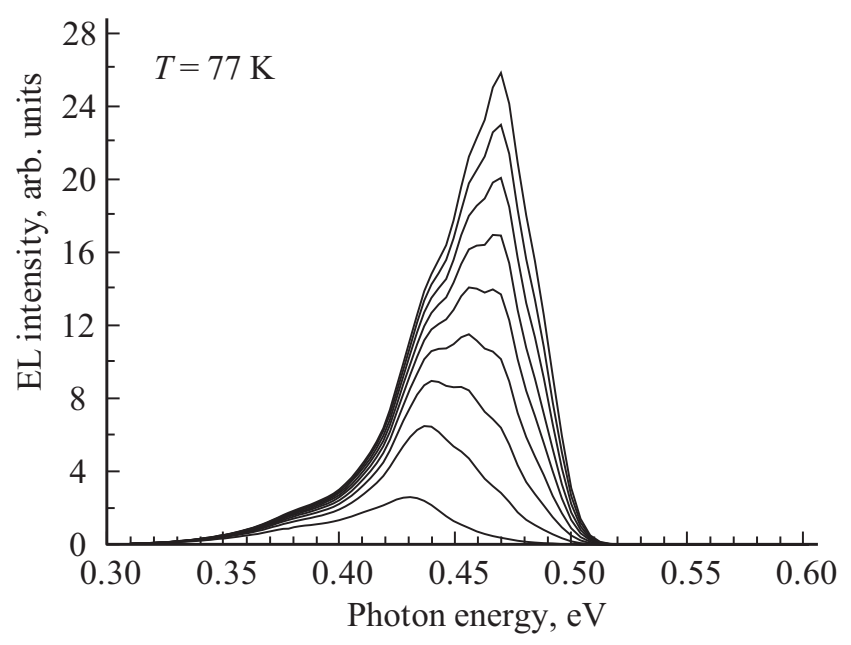

Рис. 3. Спектры ЭЛ в гетероструктуре $n^{+}$-InAs/ $n^{0}-\mathrm{InAs}_{0.59} \mathrm{Sb}_{0.16} \mathrm{P}_{0.25}$ в интервале токов накачки $20-100 \mathrm{~mA}$ с шагом $10 \mathrm{~mA}$ для случая прямого смещения при температуре $77 \mathrm{~K}$. 

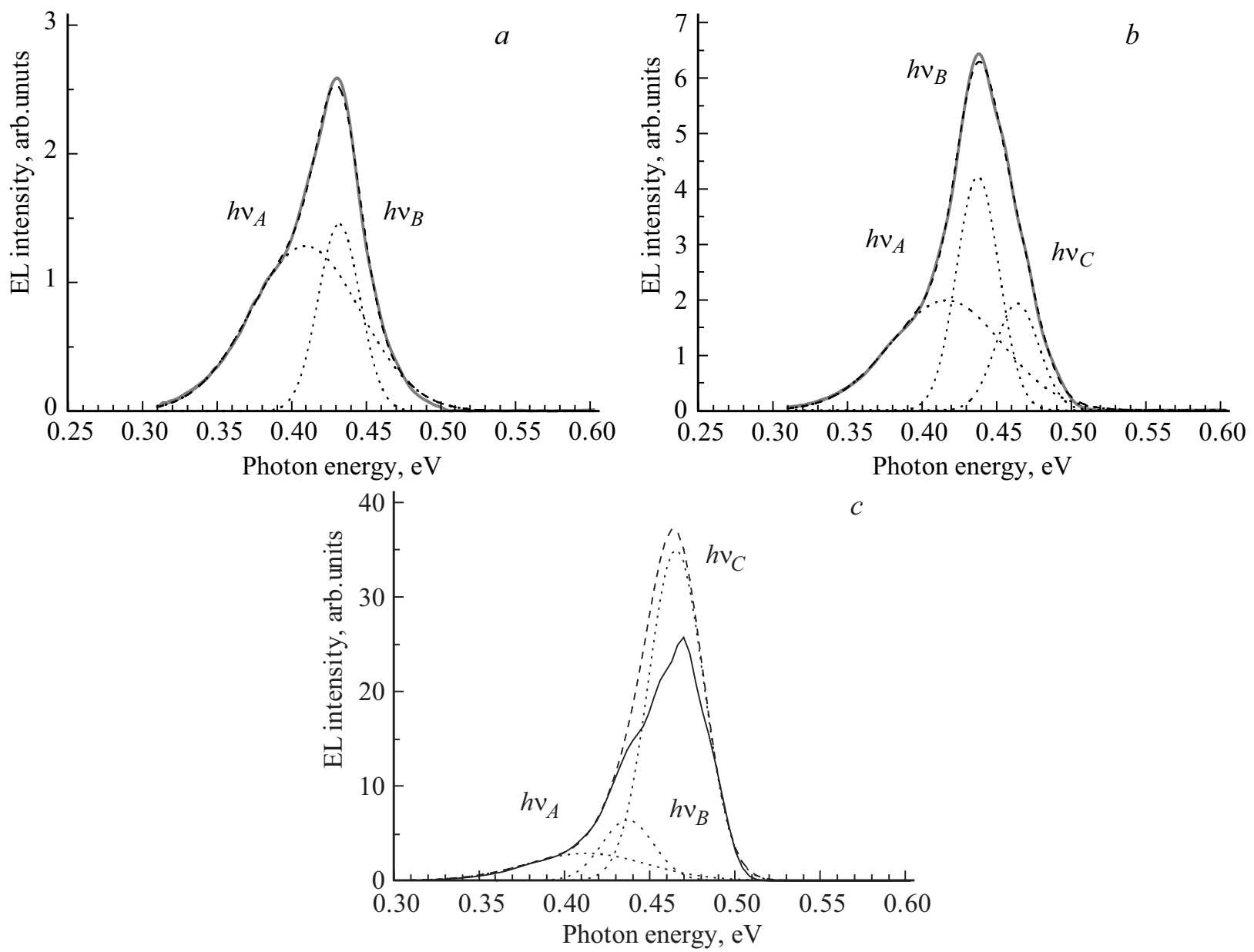

Рис. 4. Эволюция формы спектров ЭЛ в гетероструктуре $n^{+}-\operatorname{In} A s / n^{0}-\operatorname{InAs}_{0.59} \mathrm{Sb}_{0.16} \mathrm{P}_{0.25}$ при температуре $77 \mathrm{~K}$ для различных уровней накачки: $(a)-20,(b)-30,(c)-100 \mathrm{~mA}$.

$2.68 \mu \mathrm{m}, 2.78 \mu \mathrm{m}$ - углекислый газ $\left(\mathrm{CO}_{2}\right)$ [6,7]. Поэтому реальная форма спектра может быть представлена штриховой линией на рис. $4, c$, которая учитывает потери интенсивности за счет поглощения.

На рис. 5 показаны зависимости интенсивностей в максимуме излучения для результирующего спектра $\left(J_{\text {sum }}\right)$ и двух полос $h v_{B}$ и $h v_{C}$, полученных при аппроксимации экспериментальных кривых распределениями Гаусса. Соответствующая зависимость для полосы $h v_{A}$ не приведена, поскольку интенсивность данной полосы сохранялась постоянной и ее учитывали как фоновую добавку. Как видно из рис. 5, суммарная интенсивность спектра ЭЛ определяется доминирующей полосой излучения для каждой области накачки. При начальных уровнях накачки присутствовала только полоса $h v_{B}(I<30 \mathrm{~mA})$, тогда как при высоких уровнях накачки интенсивность полосы $h v_{B}$ выходила на уровень насыщения, а полоса $h v_{C}$, напротив, становилась доминирующей. Таким образом, в интервале токов 30-40 mA в исследуемой гетероструктуре наблюдается перераспределение вкладов двух различных механизмов излучательной рекомбина- ции от „интерфейсного“ к межзонному. Данный вывод согласуется с поведением ВАХ в соответствующей области смещений, прикладываемых к образцу.

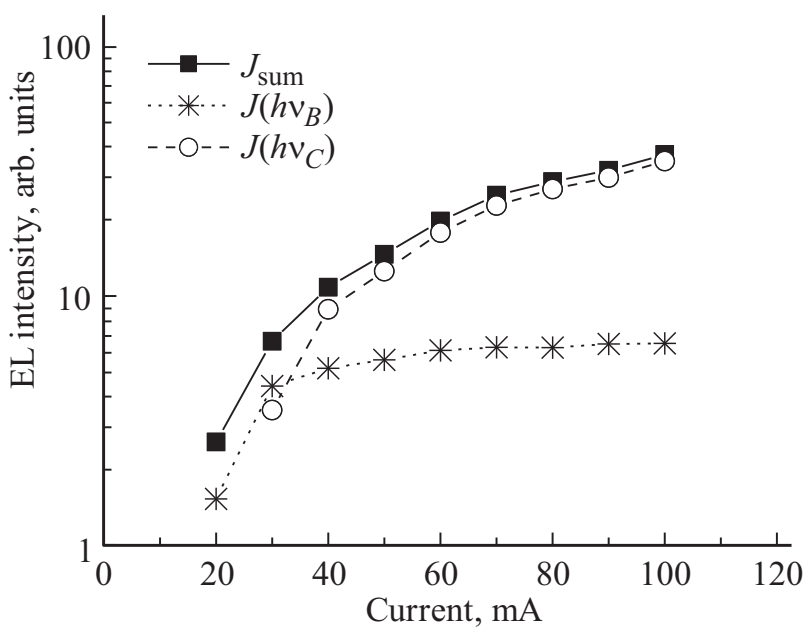

Рис. 5. Зависимость интенсивности для полос ЭЛ от тока накачки в гетероструктуре $n^{+}-\mathrm{InAs} / n^{0}-\mathrm{InAs}_{0.59} \mathrm{Sb}_{0.16} \mathrm{P}_{0.25}$ при $T=77 \mathrm{~K}$ для случая прямого смещения. 


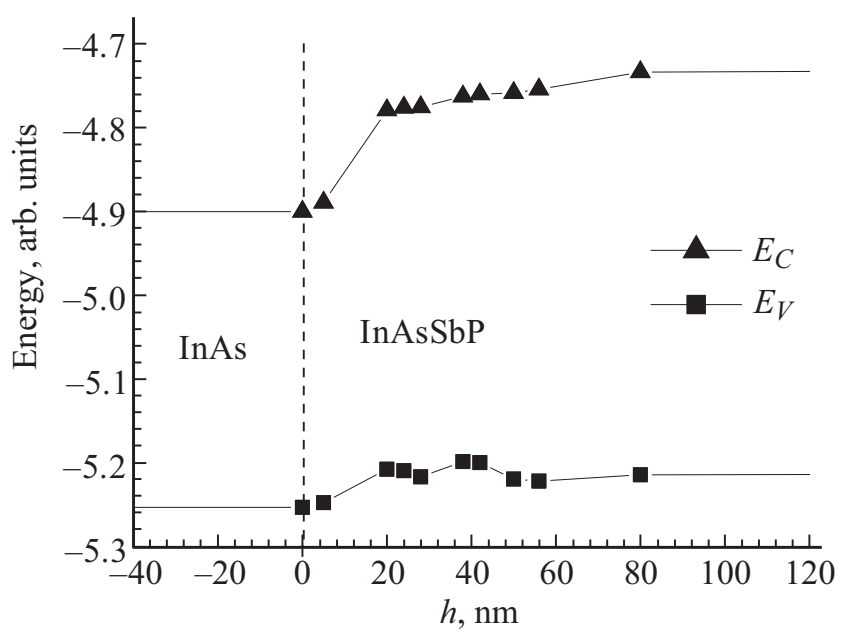

Рис. 6. Схематическая зонная диаграмма гетероперехода II типа InAs/InAs ${ }_{0.59} \mathrm{Sb}_{0.16} \mathrm{P}_{0.25}$ гетероструктуры вблизи гетерограницы при $T=300 \mathrm{~K}$. Штриховая вертикальная линия при нулевом значении $h$ обозначает границу раздела подложка/эпитаксиальный слой. В области переходного слоя показана модуляция положения потолка валентной зоны на стороне эпитаксиального слоя $\operatorname{InAs}_{0.59} \mathrm{Sb}_{0.16} \mathrm{P}_{0.25}$, рассчитанная с помощью алгоритма, использованного в работе [8].

Для начальной области ВАХ (10-40 mA) доминирующим каналом излучательной рекомбинации являются переходы носителей заряда вблизи границы раздела.

Особенность гетероструктуры II типа $n$-InAs/ $n$-InAsSbP заключается в том, что при термодинамическом равновесии в данном гетеропереходе формируются потенциальные энергетические ямы только для дырок, которые расположены на стороне эпитаксиального слоя за счет изгиба валентной зоны вблизи границы раздела. Для электронов со стороны подложки InAs на гетерогранице существует потенциальный барьер, образованный за счет энергетического скачка в зоне проводимости из-за разности величин электронного сродства арсенида индия и твердого раствора $\operatorname{InAs}_{0.59} \mathrm{Sb}_{0.16} \mathrm{P}_{0.25}$. Учитывая модуляцию состава твердого раствора в переходном слое, можно говорить о возникновении „самоорганизующихся“ потенциальных ям в валентной зоне InAsSbP, как это схематически представлено на рис. 6. Таким образом, создаются условия для локализации дырок вблизи гетерограницы в этих ямах. При приложении прямого смещения к гетероструктуре основное падение напряжения сосредоточено на стороне твердого раствора InAsSbP вблизи гетерограницы. При приложении положительного потенциала к эпитаксиальному слою происходит экстракция электронов из валентной зоны, что ведет к возникновению дырок и локализации их в квантовой яме на гетерогранице. При этом движение электронов, инжектируемых со стороны подложки $n^{+}$-InAs, ограничено потенциальным барьером на границы раздела. Тогда, при начальных уровнях накачки появляется дополнительный канал излучательной рекомбинации с энергией фотона, соответствующей эффективному энергетическому переходу с электронных состояний дна зоны проводимости на уровень локализации дырок в приграничной области порядка $60 \mathrm{~nm}$ от границы раздела $n^{+}-\operatorname{InAs} / n^{0}-\mathrm{InAs}_{0.59} \mathrm{Sb}_{0.16} \mathrm{P}_{0.25}$. Очевидно, что величина данного энергетического зазора меньше ширины запрещенной зоны объемного твердого раствора InAs ${ }_{0.59} \mathrm{Sb}_{0.16} \mathrm{P}_{0.25}$, и, по нашим оценкам, составляет $0.425 \mathrm{eV}$, что хорошо согласуется энергией фотона для полосы излучения $h v_{B}$ (рис. 4).

При увеличении внешнего смещения эффективная ширина потенциального барьера в зоне проводимости уменьшается вследствие дальнейшего увеличения изгибов зон на гетерогранице, так что данный барьер становиться туннельно-прозрачным для инжектированных электронов. Поскольку эффективность рекомбинации вблизи гетерограницы в основном определяется дырками, локализованными в квантовой яме, а их концентрация почти не меняется, то интенсивность полосы $h v_{B}$ выходит на насыщение. Электроны, прошедшие барьер на гетерогранице, излучательно рекомбинирует в объеме эпитаксиального слоя $n^{0}$-InAsSbP. C увеличением внешнего смещения, возрастает их количество, и следовательно при высоких уровнях накачки межзонные излучательные переходы вносят основной вклад в суммарную люминесценцию в исследуемой гетероструктуре, а в спектрах ЭЛ доминирует полоса $h v_{C}$, что наглядно показано на рис. 3 и 4,c. Экспериментально наблюдаемая величина энергии фотона в максимуме полосы излучения $h v_{C}(0.465 \mathrm{eV})$ несколько отличается от значения ширины запрещенной зоны $(0.49 \mathrm{eV})$ для твердого раствора InAs $\mathrm{As}_{0.59} \mathrm{Sb}_{0.16} \mathrm{P}_{0.25}$, рассчитанного с помощью алгоритма, использованного в работе [8]. Полученная разница порядка $25 \mathrm{meV}$ связана с тем, что излучательные переходы происходят между донорными и акцепторными состояниями в объеме эпитаксиального слоя.

Таким образом, была продемонстрирована перестройка спектра электролюминесценции, управляемая током накачки, протекающим через гетероструктуру. Интервал перестройки максимума излучения для данных образцов при температуре $77 \mathrm{~K}$ составил $0.3 \mu \mathrm{m}$ (от 2.9 до $2.6 \mu \mathrm{m}$ ) при изменении тока с 20 до $100 \mathrm{~mA}$. При этом существуют перспективы расширения полученного интервала еще на $0.1-0.3 \mu \mathrm{m}$ благодаря использованию дополнительных внешних факторов, таких как легирование эпитаксиального слоя акцепторной примесью, увеличение ширины запрещенной зоны твердого раствора InAsSbP за счет увеличения концентрации фосфора в твердой фазе и др. Более того, введение акцепторной примеси обеспечит не только заполнение дырками квантовых ям вблизи гетероперехода, но также существенно изменит изгибы зон на гетерогранице II типа и уменьшит энергетический зазор между уровнями локализации электронов и дырок, что отразится на спектральном положении полосы ЭЛ $h v_{B}$. 


\section{5. Заключение}

В результате проведенных исследований было показано, что в гетероструктурах на основе узкозонных полупроводников именно локализация дырок играет важную роль в реализации излучательных переходов. Особенность системы твердых растворов $\mathrm{In}-\mathrm{As}-\mathrm{Sb}-\mathrm{P}$ заключается в том, что из элементов пятой группы сурьма наиболее тяжелый по отношению к мышьяку или фосфору. Следовательно, при эпитаксиальном наращивании концентрация сурьмы в переходном слое более подтверждена флуктуациям. Известно, что в узкозонных твердых растворах на основе арсенида индия энергетическое положение потолка валентной зоны в основном определяется концентрацией атомов сурьмы [9]. Поэтому, модуляция состава эпитаксиального слоя вблизи гетерограницы выразится в флуктуации положения потолка валентной зоны и формировании потенциальных ям для дырок. Ранее в работе [10] нами было предсказано, что для эффективной локализации дырок требуется создание гораздо более высоких энергетических барьеров (в 3.4 раза выше), чем для электронов. Кроме того, пространственное разделение локализованных носителей на гетерогранице II типа позволяет существенно подавить процессы, связанные с Оже-рекомбинацией, что должно благоприятно сказаться на температурных режимах работы светоизлучающих приборов для спектрального диапазона 2-5 $\mu \mathrm{m}$. С другой стороны, при проектировании оптоэлектронных приборов, для которых тепловые потери имеют существенное значение, следует учитывать модуляцию состава эпитаксиальных слоев вблизи гетерограницы как дополнительный канал протекания управляющего тока. Для подавления модуляции состава твердого раствора в переходном слое при наращивании методом МОГФЭ необходимо подбирать соответствующие технологические условия наращивания эпитаксиальных гетероструктур.

\section{Список литературы}

[1] К.Д. Моисеев, В.В. Романов, Ю.А. Кудрявцев. ФТТ 58, 2203 (2016).

[2] В.В. Романов, М.В. Байдакова, К.Д. Моисеев. ФТП 48, 753 (2014).

[3] А. Милнс, Д. Фойхт. Гетеропереходы и переходы металлполупроводник. Мир, М. (1975). 432 с.

[4] Landolt-Börnstein. Handbook. Numerical Data. Ser. III. Springer, Berlin, Heidelberg (1982). V. 17a. P. 264; (1987), V. 22a. P. 305.

[5] А.М. Поляков, Разгаданный полупроводник. Просвещение, М. (1981). 160 c.

[6] В.В. Романов, Э.В. Иванов, А.Н. Именков, Н.М. Колчанова, К.Д. Моисеев, Н.Д. Стоянов, Ю.П. Яковлев. Письма в ЖТФ 27, 80 (2001).

[7] Р. Хадсон. Инфракрасные системы. Мир, М. (1972). 536 с.

[8] М.М. Григорьев, Э.В. Иванов, К.Д. Моисеев. ФТП 45, 1386 (2011).

[9] S.H. Wei, A. Zunger. PRB 52, 12039 (1995).

[10] Г.Г. Зегря, М.П. Михайлова, Т.И. Данилова, А.Н. Именков, К.Д. Моисеев, В.В. Шерстнев, Ю.П. Яковлев. ФТП 33, 351 (1999). 